\title{
Analysis of a delta spot
}

\author{
Y. Liu and H. Q. Zhang \\ National Astronomical Observatories, Chinese Academy of Sciences, Beijing 100012, PR China \\ Received 1 August 2001 / Accepted 30 January 2002

\begin{abstract}
. $\delta$-groups generally develop in three different ways: eruption of a single complex active region, eruption of large satellite spots near a large older spot, or collision of spots of opposite polarity from different dipoles. In this paper, we present a rare observational result in which a $\delta$-spot forms from rapid coalescence of two opposite magnetic features in a pre-existing $\delta$-configuration. The white-light (WL) observations of this $\delta$-spot in active region NOAA 9077 were performed by TRACE. The time-lapse movie of the co-aligned WL images shows that the whole active region was undergoing rapid disintegration and reconstruction on 11-17 July 2000. The preceding $(p)$ and following $(f)$ components of the $\delta$-spot continue to grow in size, while the active region is in the decaying phase. Their proper motions are determined as a function of time using SOHO/MDI full-disk magnetograms. A major flare (3B/X5.7) erupted in the active region on 14 July. Initially, a relative shearing motion is visible between the $p$ and $f$ spots on this day. About two hours before the major flare, the shearing motion suddenly stops and the velocities change significantly. The ribbons of the flare are located just outside of the $\delta$-configuration. An interesting finding is the sign reversal of the helicity of the $f$ spot also just 2 hours before the flare. The $\delta$-spot obviously separates after the flare. Our results clearly demonstrate that helicity reversal in magnetic features of a $\delta$-configuration is likely to destabilize the compact structure, as well as to re-organize the magnetic field configuration, and, hence, is important for the rapid disintegration of a $\delta$-spot during major flares. A model is presented to explain why a spot can change its chirality.
\end{abstract}

Key words. Sun: magnetic fields - Sun: sunspots - Sun: flares

\section{Introduction}

The formation and disintegration of $\delta$-configurations is an important problem in the study of the photospheric magnetic field. Using eighteen years of observations at Big Bear Solar Observatory, Zirin \& Liggett (1987) summarized that $\delta$-spots generally develop in three patterns: emergence of a single complex spot cluster, or island delta; emergence of large satellite spots very close to a large existing spot; and collision of two distinct bipolar groups. So far, it is not clear what the origin of $\delta$-spots is, and how they emerge. Although it is proposed that a $\delta$-configuration will be naturally formed if the initial twist of a magnetic tube is large enough when it emerges from below (Fan et al. 1999), there is not sufficient information about the physical cause of the separation, especially, that occurring in association with large flares.

Before recent experiments, such as SOHO and TRACE, began to operate, the observational study of the $\delta$-spots was based on their short-term morphological evolution (Tang 1983; Zirin \& Liggett 1987; Zirin 1988; Wang 1992; Shi \& Wang 1993), so that some important development stages may have been lost due to the day-night cycle

Send offprint requests to: Y. Liu,

e-mail: lyu@sun10.bao.ac.cn of the ground-based observations. Moreover, since intensive flare activity seems to be strongly connected to the $\delta$-configuration and dynamical processes (Zirin \& Tanaka 1973; Hagyard et al. 1984; Zirin \& Liggett 1987; Tanaka 1991; Schmieder et al. 1994; Li et al. 1999; Sammis et al. 2000; Liu \& Zhang 2001), a detailed analysis with observations of TRACE is desirable.

In this paper, we present a rarely observed result with TRACE $(5000 \AA)$. A $\delta$-configuration in the active region NOAA 9077 was found to form from a pre-existing one. We studied its separation and the current helicity evolution. During separation, a major flare (3B/X5.7) erupted in the active region with its ribbons at the site of the $\delta$ configuration. The period chosen for helicity measurement is over three days (13-15 July) when the active region was at the central meridian of the solar disk.

\section{Observations and data reduction}

Active region NOAA 9077 was the target of an international ground-based observing campaign at Huairou Solar Observing Station (HSOS) which was coordinated with TRACE and SOHO. A detailed analysis of the photospheric magnetograms of this active region is discussed in Liu \& Zhang (2001), who show that it is a very complex 
and highly dynamic region. Along the highly sheared magnetic neutral line, Yan et al. (2001) propose the existence of a magnetic flux rope. A description of the major flare and an associated CME is given by Zhang et al. (2001). Manoharan et al. (2001) study the interplanetary effects caused by the CME. In the present paper, we study the evolution of two small magnetic features in this region. Figure 3 shows the development of the photospheric vector magnetic field, in which the magnetic features of interest (P6 and F4) are near the magnetic neutral line. Obviously, P6 and F4 are moving toward each other and they form a $\delta$-configuration on 13 July. Because of their shearing motion, the neutral line of this active region becomes very curvy at this site. A filament is lying over the right part of the neutral line with one end also rooted at the $\delta$-configuration. Before the major flare of 14 July, the filament is activated violently. During the flare, P6 and F4 separate rapidly. P6 disappears late on 15 July while F4 remains until 17 July.

The vector magnetograph of HSOS operates at two Fraunhofer lines: FeI $\lambda 5324.19 \AA$ for photospheric and $\mathrm{H} \beta$ for chromospheric observations, respectively (Ai \& $\mathrm{Hu}$ 1986). For the photospheric magnetic field observations, the passband is tuned $-0.075 \AA$ off the FeI $\lambda 5324.19 \AA$ line center so as to measure the longitudinal component, and at the line center to measure the transverse component. In the measurement, we increase the signal-to-noise ratio by integration of 255 frames and the $4 \times 3$ average. After this, the image scale of the magnetograms is about $2^{\prime \prime}$ pixel $^{-1}$.

Some factors may cause potential problems in the calculation of current helicity with a filter-type magnetograph (Hagyard \& Pevtsov 1999; Bao et al. 2000). We have to consider Faraday rotation, magnetic saturation, and $180^{\circ}$ ambiguity. In this work, we avoid these negative effects for several reasons: (1) the observed $\delta$-configuration is of medium size and the maximum of its magnetic field is no more than $2000 \mathrm{G}$, i.e., there is no magnetic saturation in this $\delta$-configuration; (2) it is because of the median field strength in the umbrae and off-center line measurements that magneto-optical effects (Faraday rotation) are not serious (see also, Bao et al. 2000; Zhang 2000); (3) the projection effect is not important, since the active region was at the central meridian at a low latitude $\left(\mathrm{N} 17^{\circ}\right)$ during the period from 13-15 July; (4) we resolve the $180^{\circ}$ ambiguity of the transverse field by a multi-step method (Wang et al. 1994); (5) we calculate the current helicity with $B_{z}$ above $60 \mathrm{G}$ and $B_{t}$ above $200 \mathrm{G}(3 \sigma$ levels); (6) to examine the simple relation to large flares, we only emphasize the obvious variation of the helicity sign.

TRACE (Handy et al. 1999) white-light images have $1024 \times 1024$ pixel, with an $8.5^{\prime} \times 8.5^{\prime}$ field of view, $1^{\prime \prime}$ spatial resolution, and about 0.5 hour temporary resolution. The data let us trace the evolution of important spots conveniently and without ambiguity. We coaligned these images and used movie programs to show them. Readers can obtain an MPEG movie from the URL (http://sun.bao.ac.cn/staff/lyu/spot.mpe), or see it directly on line by (http://sun.bao.ac.cn/staff/ lyu/gifmovie/gifmovie.html).

We judge the spots' magnetic polarities by superimposing longitudinal magnetograms of HSOS on the corresponding white-light images. It is easy to locate one spot in a three-dimensional coordinate system and calculate its proper motion using a sequence of full-disk SOHO/MDI data and TRACE white-light images.

\section{Formation of $\delta$-spot P6-F4}

AR 9077 was in a highly sheared magnetic configuration classified as $\beta \gamma \delta$. Here, we follow the nomenclature in Liu $\&$ Zhang (2001) to describe the spots. Figure 1 shows some images illustrating the evolution of the global spot group. The main spots are P1, P2, and F1 on 11 July. P1 and F1 are in a large $\delta$-structure when the active region first emerges from the solar east limb. Many small pores, including F4 and P6, are growing and coalescing in their penumbrae as evident in the white-light movie. On 11 July, $\mathrm{F} 4$ is in the south of the penumbra of $\mathrm{P} 1$, so that they are also in a local $\delta$-configuration, although their areas and fluxes are not balanced.

It is interesting that F4 is "dropped" by the fast westmoving $\mathrm{P} 1$ and met by $\mathrm{P} 6$ to form a new small $\delta$-spot early on 13 July. The $\delta$-spot P6-F4 grows quickly in size and separates from the old spot, developing a new penumbra. Since the formation of $\delta$-spot P6-F4 is related to the old delta structure, it can be classified as a special case of those listed in Sect. 1. On 14 July, a major 3B/X5.7 flare erupts at 10:10 UT. About two hours before, P6-F4 begin an obvious and quick separation with $\mathrm{P} 6$ sliding to the north of F4. The next day, $\mathrm{P} 6$ disappears and F4 coalesces with the penumbra of a new spot P9 which is indicated by an arrow in Fig. 1.

Figure 2 outlines the location of P6-F4 in the active region on 14 July and gives an enlargement of this region with vector magnetic field overlaying the white-light image. Detailed magnetic field evolution is shown in Fig. 3. The $\delta$-configuration of P6-F4 exists from 13-15 July. The major flare seems to be the accelerator of its separation. Three boxes shown in Fig. 3 indicate the $\delta$-configurations formed by F4 with P1, P6 and P9, respectively. This implies that F4 is a very dynamic magnetic feature worth deep research.

\section{Motions of P6-F4 on 14 July}

Many observations show that during a large flare the position and shape of a sunspot can be obviously perturbed. Proper motion calculations are important for understanding the relation between spots dynamics and flares. Because of the limits of ground-based observations, such as seeing, intermission, precision, and instrumental resolution, it is difficult to precisely measure the proper motions. Using the YOHKOH white-light data, Anwar et al. (1993) find a rapid sunspot displacement for a whitelight flare (X1.5). The approximate velocity is as high as 


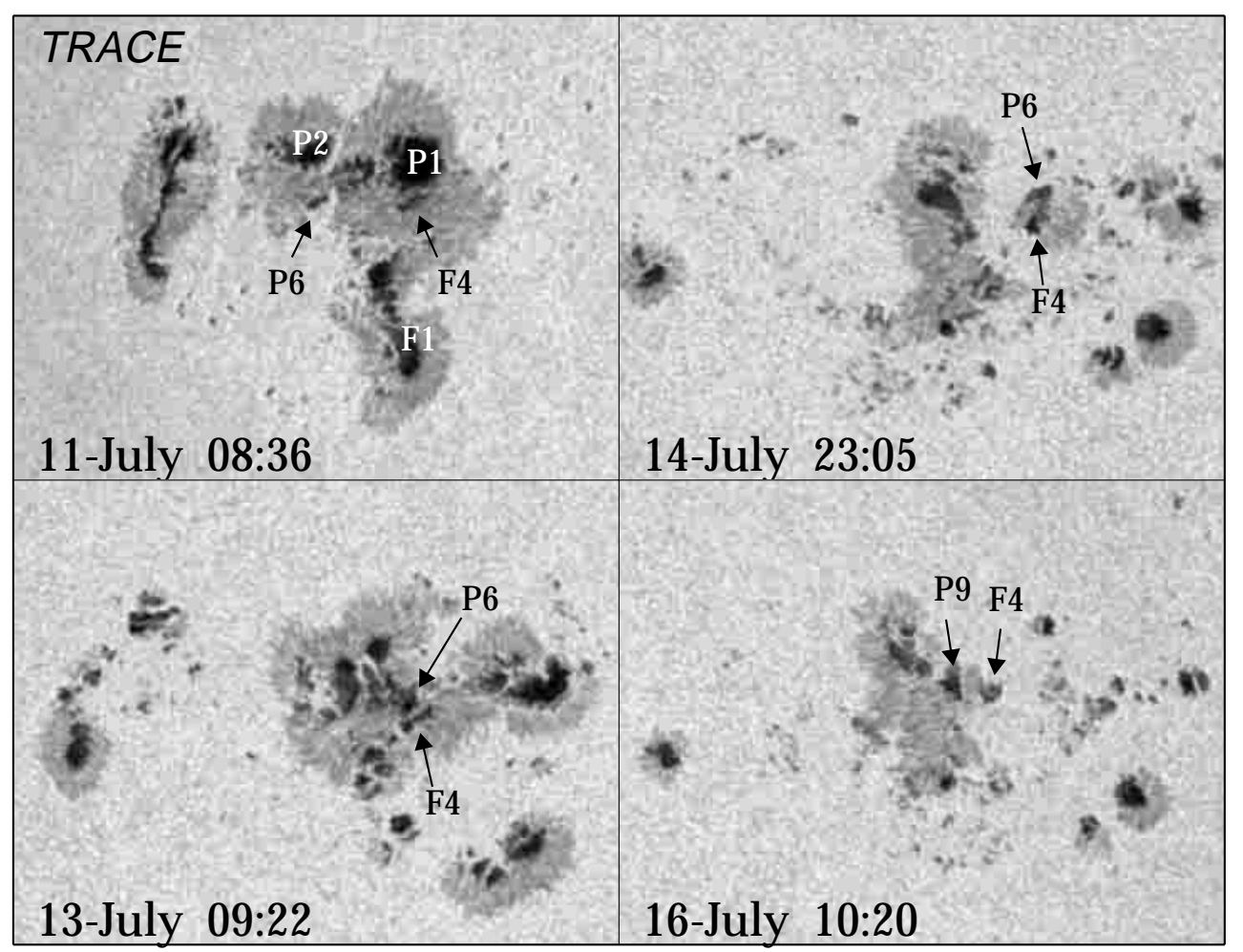

Fig. 1. TRACE white-light $(5000 \AA)$ images of the evolution of sunspot group AR 9077. The main spots are P1, P2 and F1 on July 11, 2000. Spots F4 and P6 moved together to form a $\delta$-spot directly from different penumbrae on July 13. "P" means preceding (positive), and "F" following (negative). FOV: $3^{\prime} \times 2.3^{\prime} .($ An MPEG movie can be obtained from the URL http://sun.bao.ac.cn/staff/lyu/spot.mpe, or seen directly on-line at http://sun.bao.ac.cn/staff/lyu/gifmovie/gifmovie.html)

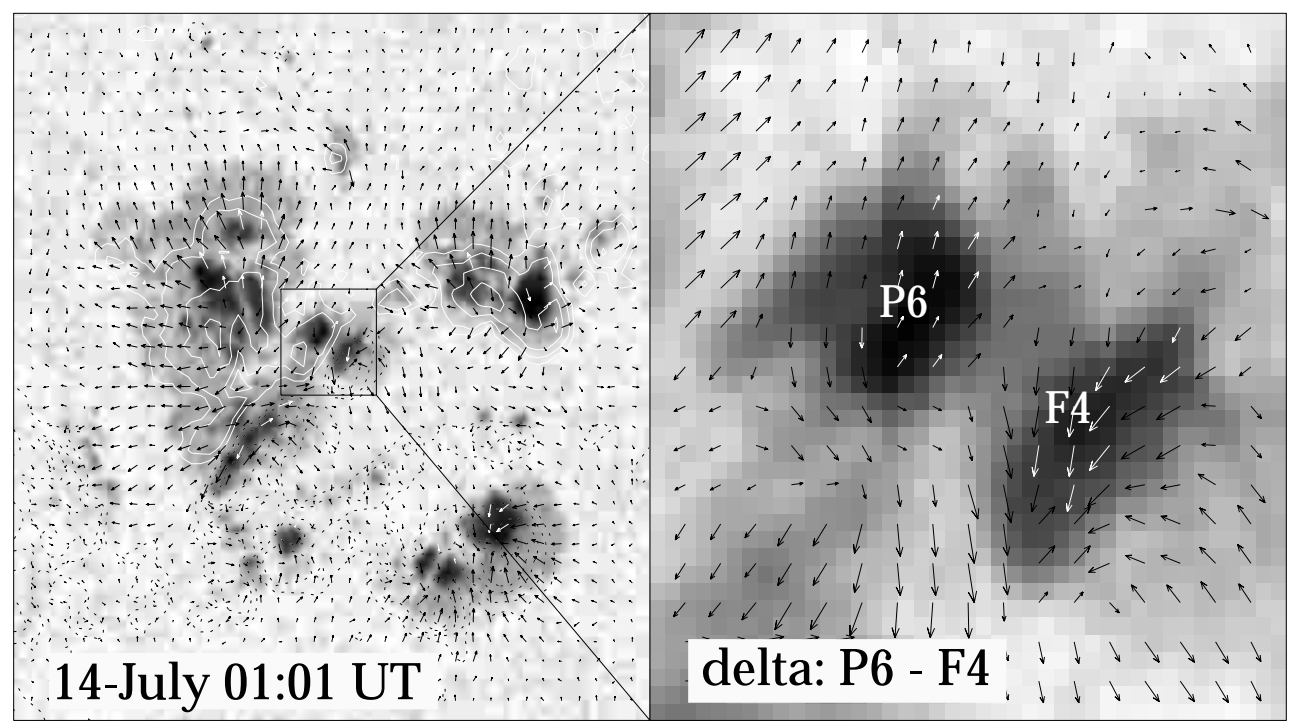

Fig. 2. Vector magnetogram of HSOS superimposed on the corresponding white-light image from TRACE. The white contours represent positive longitudinal magnetic fields, black contours represent negative fields. Arrows are in the direction of the transverse fields with a length proportional to the field strength. The box in the left frame outlines the $\delta$-spot P6-F4. The right frame is an enlargement with transverse fields superimposed. The magnetic contour levels are 600, 1200, 2000, and 3000 G. The FOV of left frame is $2.1^{\prime} \times 2.1^{\prime}$ and $20^{\prime \prime} \times 20^{\prime \prime}$ for the right frame.

$2.0 \mathrm{~km} \mathrm{~s}^{-1}$. The major flare of 14 July is also a whitelight flare (X5.7, 10:10 UT) with two ribbons just outside of F4 and P6. For this reason, we study the proper motions on 14 July. The proper motion measurements are based on the method described by Liu \& Zhang (2001) and the positions are translated into Carrington coordinates. Figure 4 displays the projections of the trajectories into the Carrington system with a time interval of 96 minutes. 

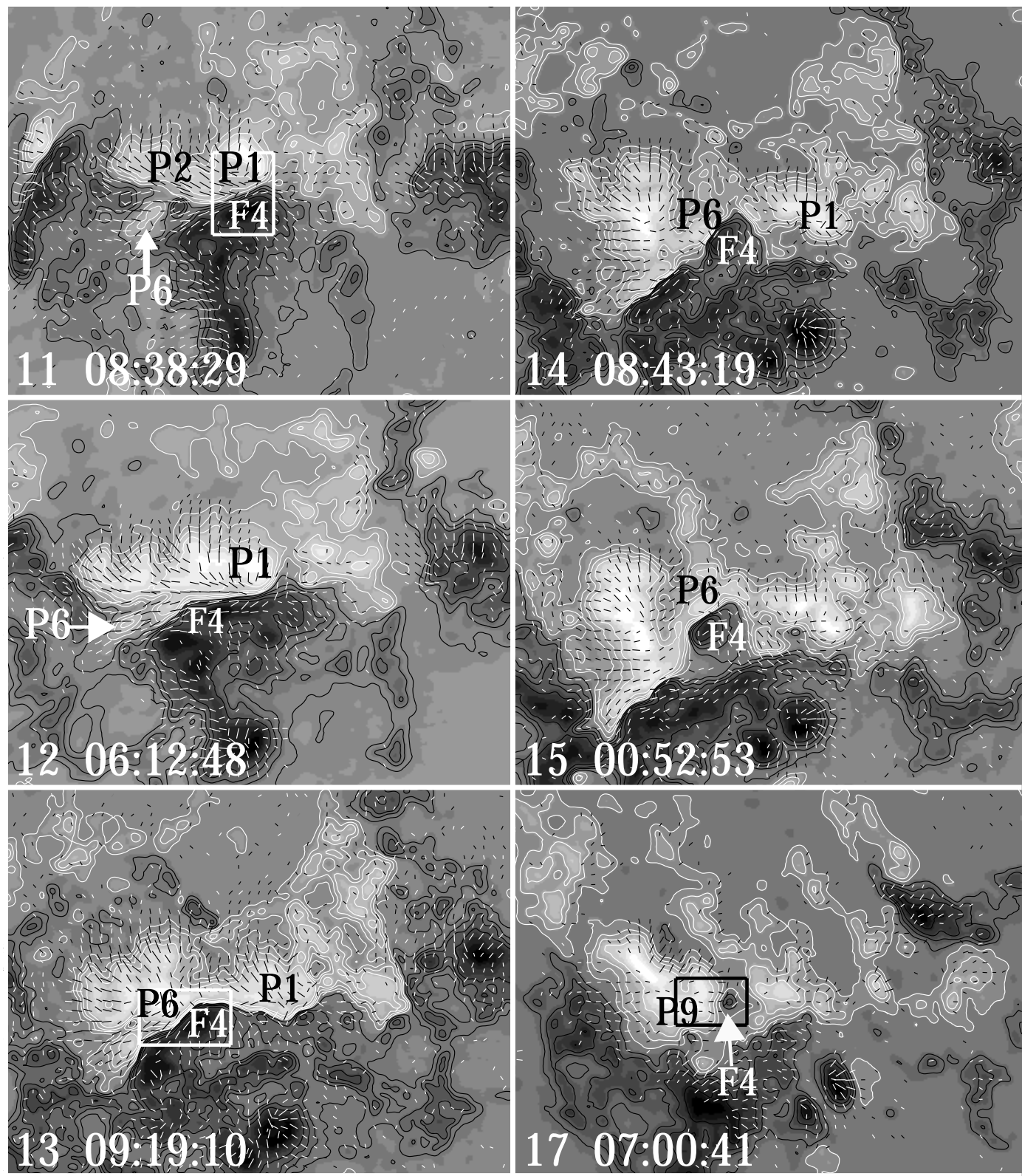

Fig. 3. A sequence of vector magnetograms observed from 11 to 17 July. FOV: $3^{\prime} \times 2.3^{\prime}$. White (black) patches are positive (negative) longitudinal magnetic fields. The contour levels of the longitudinal field are 160, 420, 680, and 1070 G. White contours represent positive polarities and black contours negative polarities. The bars indicate transverse fields with a length proportional to the field strength. Note, that the three boxes are at the locations of the $\delta$-configurations formed by F4 with P1, P6, and P9, respectively.

In this figure, the arrowheads and black points represent their positions with time. It is obvious that there are two different periods: (a) before 08:00 UT, we observe relative shear motions between P6 and F4 and (b) after 08:00 UT, we find irregular motions between them.

Based in Fig. 4, the velocities are calculated and shown in Fig. 5. F4 is represented by squares while P6 by diamonds. The dashed lines indicate the time of the flare maximum (10:24 UT). The spots do not show as high a rate as that found by Anwar et al. (1993). Obviously, about two hours before the flare, F4 reaches its highest velocity $\left(0.73 \mathrm{~km} \mathrm{~s}^{-1}\right)$ while P6 slows down to its lowest velocity $\left(0.03 \mathrm{~km} \mathrm{~s}^{-1}\right)$. Around the flare maximum, both of them move at a normal rate. Therefore, it seems that two hours before the major flare, the $\delta$-configuration is disturbed. Note that, after the flare maximum, P6 slows down again to a very low velocity for its decay. P6 disappears completely late on the next day. There are no X-class flares in the super-active region after 14 July. We also calculate the proper motions of $\mathrm{P} 6$ and $\mathrm{F} 4$ for 12 hours after the flare, but find no abnormality.

\section{Current helicity evolution}

NOAA 9077 is at the central meridian on 14 July. HSOS observed this active region and obtained vector magnetic 
Table 1. List of magnetograms in active region 9077. Column $h_{\mathrm{c}}$ gives the helicity sign of spot F4.

\begin{tabular}{|c|c|c|c|c|c|c|c|}
\hline No. & $\begin{array}{l}\text { Time } \\
\text { (UT) }\end{array}$ & $\begin{array}{l}\text { Date } \\
\text { (July) }\end{array}$ & $\begin{array}{l}h_{\mathrm{c}} \\
(\mathrm{F} 4)\end{array}$ & No. & $\begin{array}{l}\text { Time } \\
\text { (UT) }\end{array}$ & $\begin{array}{l}\text { Date } \\
\text { (July) }\end{array}$ & $\begin{array}{l}h_{\mathrm{c}} \\
(\mathrm{F} 4)\end{array}$ \\
\hline 1. & 09:19 & 13 & - & 10. & $05: 53$ & & + \\
\hline 2. & $22: 58$ & & - & 11. & $06: 34$ & 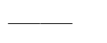 & - \\
\hline 3. & $23: 31$ & 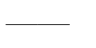 & - & 12. & 07:08 & 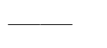 & - \\
\hline 4. & 00:08 & 14 & - & 13. & $07: 59$ & & - \\
\hline 5. & 01:01 & & - & 14. & $08: 12$ & & - \\
\hline 6. & 01:19 & & - & 15. & $08: 43$ & & + \\
\hline 7. & $03: 27$ & $\longrightarrow$ & - & 16. & $08: 55$ & 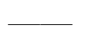 & + \\
\hline 8. & $04: 14$ & $\longrightarrow$ & - & 17. & 09:42 & . & + \\
\hline 9. & $04: 47$ & $\square$ & - & 18. & 00:52 & 15 & + \\
\hline
\end{tabular}

field data during good seeing conditions. We choose 18 magnetograms (13-15 July, listed in Table 1) for the study of the $\delta$-configuration P6-F4. Because the active region is very close to the disk center, we assume that the longitudinal field $B_{\|}$can be approximated by $B_{z}$. In the data reduction, we avoid the negative effects listed in Sect. 2 for the reasons stated there. The vertical current density is calculated by

$J_{z}=\frac{1}{\mu_{0}}\left(\frac{\partial B_{y}}{\partial x}-\frac{\partial B_{x}}{\partial y}\right)$

with the differencing method for the filtered transverse field measurement.

We define the density of vertical current helicity as

$h_{\mathrm{c}}=B_{z} \cdot J_{z}$.

It is a measurable component of the photospheric field and has been widely used in helicity research (e.g., Bao \& Zhang 1998).

Figure 6 shows the evolution of the current density for P6-F4 over three days. The positive polarity of the magnetic field is given in white, while the negative is given in black. Solid contours represent upward components of current density, while dashed represent downward components. The levels of current density are $\pm 2,8$, and $20 \mathrm{Am}^{-2}$. Positions of $\mathrm{P} 6$ and $\mathrm{F} 4$ are also precisely marked in this figure.

We find an obvious current reversing process for F4. On 13 July, F4 is dominated by a large, positive current patch (its helicity is negative and the same as the predominant, left-handed twist of the northern hemisphere). On 14 July, the current density of F4 is decreased and the patch separates from its environment. Finally, its current density becomes negative on 15 July. During the three days, the current of $\mathrm{P} 6$ retains the same negative polarity. From Eq. (2) we can see that if the sign of the average vertical current is reversed then the sign of the chirality (current helicity) will also be reversed. We also demonstrate the process of helicity evolution in Table 1 and Fig. 7. In Fig. 7, F4 and P6 are shown in the top two panels, the total active region and the $\delta$-configuration of F4-P6 are shown in the bottom two panels. The helicity

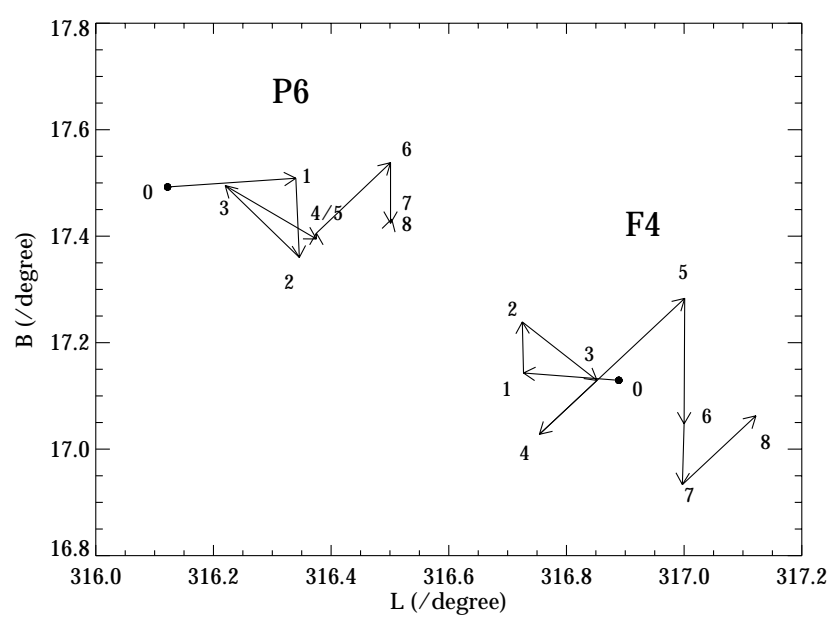

Fig. 4. Proper motions of F4 and P6 on 14 July 2000. Arrowheads and black points represent positions with time, the coordinates are in the Carrington system. "0" - 23:59, "1" 01:39, "2" - 03:15, "3" - 04:51, "4" - 06:24, "5" - 08:00, "6" $09: 36, " 7 "-11: 12$, and "8" - 12:48.
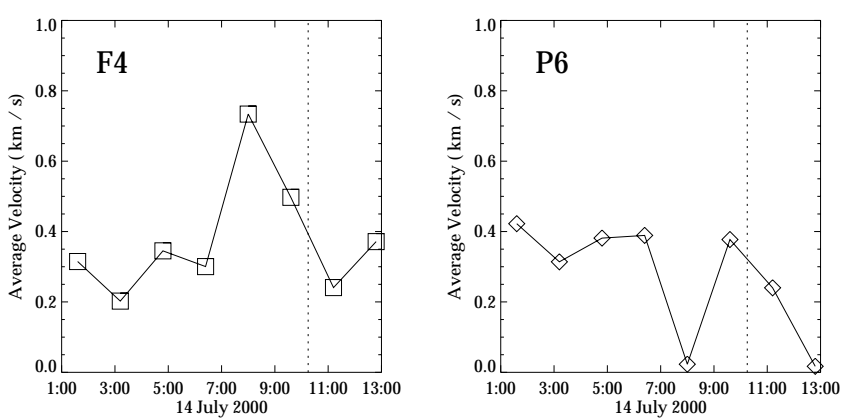

Fig. 5. Velocity of spots F4 and P6 as a function of time. F4 is denoted by squares and P6 by diamonds. The left panel shows the velocity of F4 on 14 July and the right panel corresponds to P6. The dashed lines indicate the time of the major flare at its maximum (10:24 UT). The errors are smaller than the sizes of the squares and the diamonds.

density of NOAA 9077 varies over a small range from -2 to $-7 \times 10^{-3} \mathrm{G}^{2} \mathrm{~m}^{-1}$, while the other three objects (F4, $\mathrm{P} 6$, and $\mathrm{F} 4-\mathrm{P} 6$ ) fluctuate over wider ranges from near zero to more than $-100 \times 10^{-3} \mathrm{G}^{2} \mathrm{~m}^{-1}$. At 05:53 UT on 14 July, F4 reverses the helicity sign once (see Table 1), but returns to its original state in the next magnetogram. This may be due to the onset of strong disturbance. Between 08:12 UT and 08:43 UT, about two hours before the major flare, F4 alters irreversibly its chirality.

\section{Discussion and conclusions}

TRACE white-light images present a $\delta$ spot which is formed by rapid coalescence of two magnetic features in a pre-existing $\delta$-configuration in NOAA 9077 . The origin of this $\delta$ spot is rather different from the regular patterns described by Zirin \& Liggett (1987). Particularities of this $\delta$-spot have been shown in some papers. This $\delta$ configuration is found to be the position of one end of a rising magnetic flux rope system (Yan et al. 2001), or of 


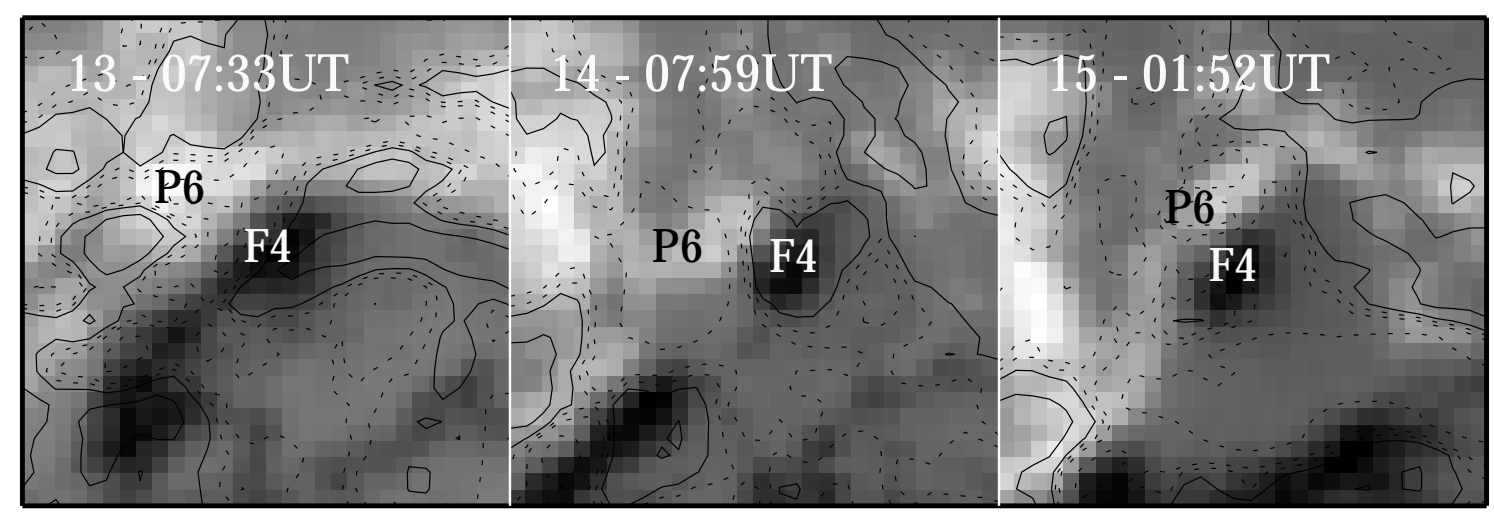

Fig. 6. Evolution of vertical current density over three days. The positive polarity magnetic field is in white, while the negative is in black. The solid contours represent upward components of current density, while the dashed represent downward components, their levels are $\pm 2,8$, and $20 \mathrm{Am}^{-2}$. P6 and F4 are marked on their locations precisely in the figure. The FOV is $0.9^{\prime} \times 0.9^{\prime}$ and the differences in the gray level of the magnetic field are due to seeing. Note the current evolution in the neighborhood of F4.
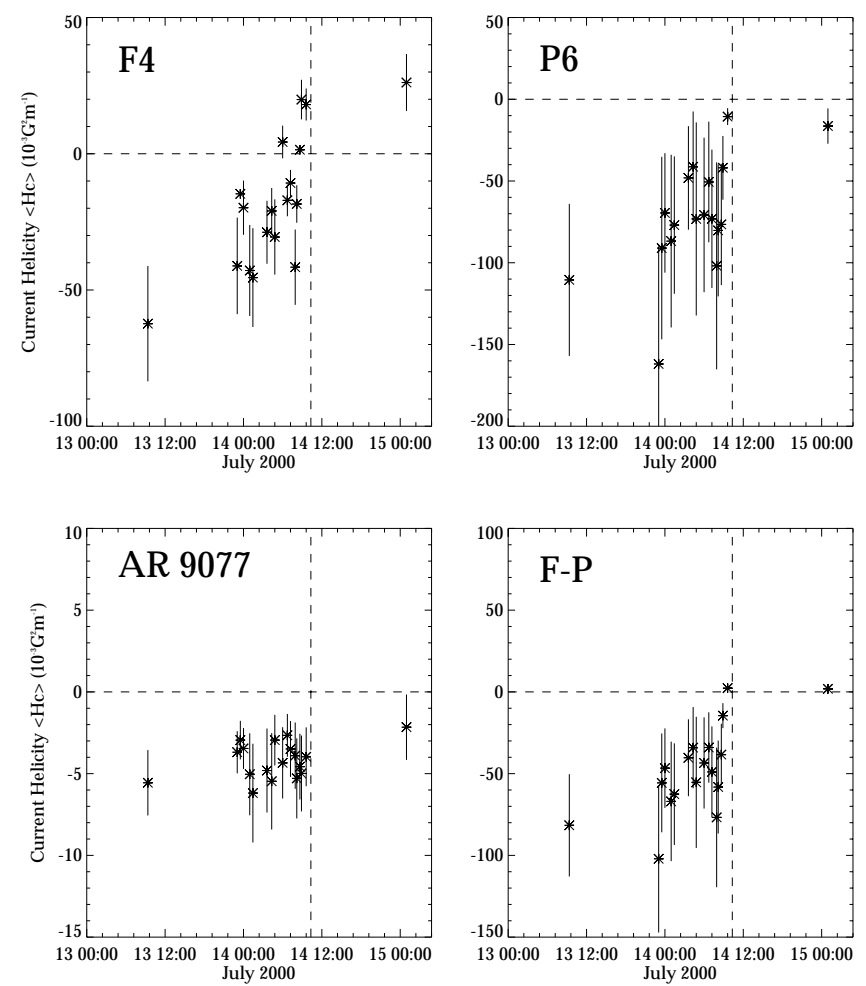

Fig. 7. Measurement of $h_{\mathrm{c}}$ based on 18 magnetograms for three successive days for AR 9077. One asterisk represents one magnetogram. Error bars reflect the variation in $h_{\mathrm{c}}$ from independent measurements. The top two panels are the results of spots F4 and P6, respectively. The bottom two panels are the total active region and total $\delta$-spot F4-P6, respectively. The vertical dashed lines represent the time of the flare maximum and the horizontal lines represent to zero helicity.

an activated filament (Zhang et al. 2001). Kosovichev \& Zharkova (2001) find that it is a magnetic transient region during the major flare.

In the present work, we find that $\delta$-configuration $\mathrm{P} 6$ F4 is important for the formation of the highly sheared magnetic neutral line of NOAA 9077, and, hence for the trigger of the filament activation and an associated CME.

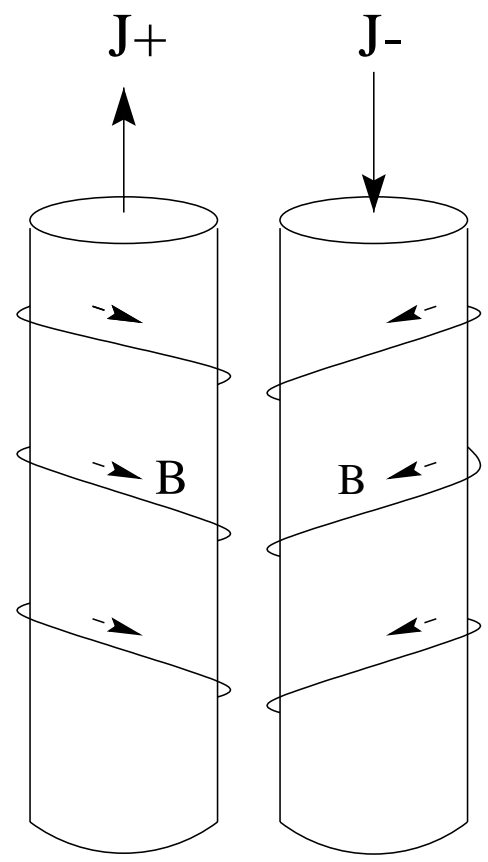

Fig. 8. Magnetic flux loop model. Two such systems could coexist in a spot. No reconnection happens between them. Note that the induced currents are opposite to each other.

Almost all of the obvious changes (motions, velocities, and current helicity) of the $\delta$-configuration developed about two hours before the major flare.

Undoubtedly, magnetic chirality (handedness) plays an important role in reconnection. In the corona, shorter Xray loops with the same chirality tend to coalesce and form longer forward or backward sigmoids (Canfield \& Pevtsov 1996; Pevtsov \& Canfield 1996). In the chromosphere, two close filaments may unite into a single one if their axial fields are in the same direction (Martin et al. 1994). In the photosphere, two spots of the same chirality can form a $\delta$-spot under the third pattern mentioned in Sect. 1. Certainly, if the helicity of one spot is reversed for some reason then it is hard for the $\delta$-configuration to keep its compact configuration. Therefore, separation will be seen. 
Based on the observational results, we think that the disintegration of $\mathrm{P} 6-\mathrm{F} 4$ is caused by the sign reversal of the current helicity of F4, while the major flare accelerates the separation. To explain the reversal of helicity, we introduce a magnetic flux loop model for spots. In Fig. 8, two twisted loop systems co-exist in the magnetic system of a sunspot, which is not in contradiction to observations and theory (Pevtsov et al. 1994; Stenflo et al. 1984). The currents induced by the loops shown in Fig. 8 should be in opposite directions. There will be no reconnection at the interface between these loops because of the identical directions of their field lines. Possible reconnection between twisted loop systems are discussed in Sakai \& Koide (1992).

If this is the case, let us consider the process of current reversal for F4. Originally, a larger portion of the left-handed helicity loop system (left one in Fig. 8) exists in F4. Two hours before the major flare, some disturbance, which cannot be derived from our observation, produces the dominance of the right-hand helicity (right one in Fig. 8). Thus, the average current of F4 is reversed and is now negative.

In contrast to our previous work, we study for the first time the relation between helicity evolution and $\delta$ configurations. In future work, it is essential to study more complex $\delta$-configurations and examine whether it is a universal rule that $\delta$-spots can be disintegrated by helicity reversal.

Acknowledgements. We would like to thank Dr. C. Denker for very helpful comments that improved the manuscript. The vector magnetic field data are provided by Huairou Solar Observing Station. The white light data are provided by the TRACE team. TRACE is a NASA Small Explorer project. We thank the SOHO/MDI team for the full-disk MDI magnetograms. This work was supported by the National Natural Science Foundation of China under grants No. 19791090 and 10073013.

\section{References}

Ai, G., \& Hu, Y. 1986, Publ. Beijing Astron. Obs., 8, 1
Anwar, B., Acton, L. W., Hudson, H. S., et al. 1993, Sol. Phys., 147, 287

Bao, S. D., Pevtsov, A. A., Wang, T. J., \& Zhang, H. Q. 2000, Sol. Phys., 195, 75

Bao, S. D., \& Zhang, H. Q. 1998, ApJ, 496, L98

Canfield, R., \& Pevtsov, A. 1996, ASP Conf. Ser., 111, 341

Fan, Y., Zweibel, E. G., Linton, M. G., \& Fisher, G. H. 1999, ApJ, 521, 460

Hagyard, M. J., \& Pevtsov, A. A. 1999, Sol. Phys., 189, 25

Hagyard, M. J., Smith, J. B. Jr., Teuber, D., \& West, E. A. 1984, Sol. Phys., 91, 115

Handy, B. N., Acton, L. W., Kankelborg, C. C., et al. 1999, Sol. Phys., 187, 229

Kosovichev, A. G., \& Zharkova, V. V. 2001, ApJ, 550, L105

Li, W., Chik-Yin, Lee, Chae, J., et al. 1999, Magnetic Fields and Solar Processes, ed. A. Wilson, ESA SP Ser., 448, 169

Liu, Y., \& Zhang, H. 2001, A\&A, 372, 1019

Manoharan, P. K., Tokumaru, M., Pick, M., et al. 2001, ApJ, 559, 1180

Martin, S. F., Bilinioria, R., \& Tlacadas, P. W. 1994, Solar Surface Magnetism, ed. R. J. Rutten, \& C. J. Schrijver, 303

Pevtsov, A. A., \& Canfield, R. C. 1996, ApJ, 473, 533

Pevtsov, A. A., Canfield, R. C., \& Metcalf, T. R. 1994, ApJ, 425, L117

Sakai, J., \& Koide, S. 1992, Sol. Phys., 142, 399

Sammis, I., Tang, F., \& Zirin, H. 2000, ApJ, 540, 583

Schmieder, B., Hagyard, M. J., Ai, G., et al. 1994, Sol. Phys., 150, 199

Shi, Z., \& Wang, J. 1993, Sol. Phys., 149, 105

Stenflo, J. O., Harvey, J. W., Brault, J. W., \& Solanki, S. 1984, A\&A, 131, 333

Tanaka, K. 1991, Sol. Phys., 136, 133

Tang, F. 1983, Sol. Phys., 89, 43

Wang, H. M. 1992, The Solar Cycle, ASP Conf. Ser., 27, 97

Wang, T., Xu, A., \& Zhang, H. 1994, Sol. Phys., 155, 99

Yan, Y., Deng, Y., Karlicky, M., et al. 2001, ApJ, 551, L115

Zhang, H. 2000, Sol. Phys., 197, 251

Zhang, J., Wang, J., Deng, Y., et al. 2001, ApJ, 548, L99

Zirin, H. 1988, Astrophysics of the Sun (Cambridge Univ. Press)

Zirin, H., \& Liggett, M. A. 1987, Sol. Phys., 113, 267

Zirin, H., \& Tanaka, K. 1973, Sol. Phys., 32, 173 